Fate did not permit Ilting to complete his commentary on Hegel's philosophy of right which was to be volume $\mathrm{V}$ of his monumental Rechtsphilosophie-Edition; large parts of it remain still in manuscript. This was to be a part of the grand project, on which llting worked to the very end, viz. the systematic establishment and development of a practical philosophy on the basis of a critical reconstruction of past text.

Hansgeorg Hoppe

University of the Saarland

\title{
Bibliography of K.-H. Ilting's Works
}

A. Books, essays and articles.

I. Das Problem einer phänomenologischen Anthropologie, Diss. Bonn 1949.

2. 'Metaphysik und Seinsgeschichte', Philos. Rundschau 8, I960, 233-26I.

3. 'Sein als Bewegtheit', Philos. Rundschau 10, 1962, $31-49$.

4. Platons Theorie der Wirklichkeit, Habil-Schrift Kicl 1962.

5. 'Hegels Auseinandersetzung mit der aristotelischen Politik', Philos. Jahrbuch der Görres-Gesellschaft 71, 1963, 38-58.

6. 'Hobbes und die praktische Philosophie der Neuzeit', Philos. Jahrbuch der GörresGesellschaft 72, 1964, 84-102.

7. 'Zur Philosophie der Pythagoreer', Archiv f. Bcgriffsgeschichte 9, 1964, 103-131.

8. 'Aristoteles über Platons philosophische Entwicklung', Zcitschr. f. philos. Forschung XIX, 1965, 379-392.

9. 'Die Parteien im demokratischen Staat', Speculum 4, 1967, II-13.

10. 'Uber die Sprache des Naturrechts', in: Das Problem der Sprache (VII. Deutscher Kongreß für Philosophie), hrsg. v. H.G. Gadamer, München 1967, 335-350.

11. 'Die "Ungeschriebenen Lehren" Platons: Der Vortrag "Über das Gute", Phronesis 13, 1968, 1-31.

12. 'The structure of Hegel's philosophy of right', in: Hegel's political philosophy, ed. Z.A. Pclczynski, Cambridge I97ı, 90*1 10.

13. 'Naturalistic fallacy in Kant', in: Procecdings of the Third International Kant Congress, ed. L.W. Beck, Dordrecht 1972.

14. 'Anerkennung. Zur Rechtfertigung praktischer Sätze', in: G.G. Gram (Hrsg.), 
Probleme der Ethik, Freiburg 1972, 63-107. Wiederabdruck in: Rehabilitierung der praktischen Philosophie, Bd. 2, hrsg. v. M. Riedel, Freiburg/München 1972. 15. 'A porie', in: Handbuch philosophischer Grundbegriffe, München 1973, $110-118$. 16. 'Hegels Politiske Posisjon', Restaurasjonstiden, Oslo 1974.

17. 'Zur Dialektik in der Rechtsphilosophie', in: Hegel-Jahrbuch 1975, 36-44.

18. 'Geltung als Konsens', in: Ncue Hefte für Philosophic, Heft 10, Göttingen 1976, 20-5o.

19. Hegel diverso. Le filosofie del diritto del I 8 I 8 al I 83 I, Bari: Laterze 1977. XII, 240 pp.

20. 'Hegels Begriff des Staates und die Kritik des jungen Marx', in: Rivista di Filosofia, Turin 1977, 116-168.

21. 'Antike und moderne Ethik', in: Gymnasium XXIV, 1977, 149-167.

22. 'Bedürfnis und Norm. Platons Begründung der Ethik', in: Vernünftiges Denken, hrsg. v. J. Mittelstraß/M. Ricdel, Berlin/New York 1978, 420-446.

23. 'Naturrecht', in: Geschichtliche Grundbegriffe IV. hrsg. v. Brunner/Conze/Koselleck, Stuttgart 1978, 245-313.

24. 'Wahrheit und Verbindlichkeit", in: Konstruktionen versus Positionen, hrsg. v. K. Lorenz, Berlin 1979, $115-145$.

25. "La forme logique et systématique de la Philosophie du droit", in: Hegel ct la philosophie du droit, Paris: Presses Universitaires de France 1979, 35-64.

26. 'Tecnica e Prassi in Heidegger e Marx', in: Metaphorcin, Napoli 1979, 100-108.

27. 'Gibt es eine kritische Ethik und Rechtsphilosophic Kants?', Archiv. f. Gesch. d. Philos., Bd. 63.3, 1981, 325-345.

28. 'Ontologie, Metaphysik und Logik in Hegcls Erörterung der Reflexionsbestimmungen", Revue Internationale de Philosophic, No. I 39-140, $162,95-110$.

29. 'Rechtsphilosophic als Phänomenologie des Bewußtseins der Freiheit', in: Hegels Philosophie des Rechts. Die Theorie der Rechtsformen und ihre Logik, hrsg. v. D. Henrich/R.P. Horstmann (Veröffentlichung der Internationalen HegelVereinigung), Stuttgart 1982, 225-254.

30. 'Sittlichkeit und Höflichkeit. Oder: Von der Würde der Persönlichkeit und der Verletzlichkeit des Amour Propre', in: Aspekte der Kultursoziologie. Festschrift für Mohammed Rassem, hrsg. v. J. Stagl, Berlin 1982, 99-121.

3r. 'Der Geltungsgrund moralischer Normen', in: Kommunikation und Reflexion.

Festschrift für Hans-Otto Apcl, hrsg. v. Wolfgang Kuhlmann und Dictrich Bochler, Suhrkamp, Frankfurt/M 1982, 612-648.

32. 'Herrschaft', in: Geschichtliche Grundbegriffe, Bd. 3, H-Mc, hrsg. v. Brunner/Conze/Koselleck, Klett-Cotta, Stuttgart 1982, 33-39.

33. 'Macht, Gewalt', in: ditto, 854-865, 888-808, 925929 .

34. 'Universidalidad e historicidad de las normas práticas', in: Compilacion de Comunicaciones, Volumen $"$ (Primer Congreso Internacional de Filosofia del Derecho, 19 al 23 de Octubre de 1982, La Plata, Argentina), 593-606. 
35. 'Il concetto hegeliano di filosofia della natura', in: Giornale di Metafisica (Nuova Seric), IV, Genova 1982, $297\{-306$.

36. Naturrecht und Sittlichkeit. Begriffsgeschichtliche Studien (Sprache und Geschichte, Band 7, hrsg. v. R. Koselleck und K. Sticrle). Klett-Cotta, Stuttgart $1983,304 \mathrm{pp}$.

37. 'L'uomo e la natura nel pensiero hegeliano', Il Tempo, 4 Gennaio 1984, 3.

38. 'Hegel's concept of the state and Marx's early critique', in: The State and Civil Socicty. Studies in Hegel's Political Philosophy. Edited by Z.A. Pelczynski, Cambridge $1984,93-113$.

39. 'The dialectic of civil socicty', in: ditto, 2 I I-226.

\section{B. Editions and translations}

1. Ferdinand Tönnies: Thomas Hobbes. Leben und Lehre. Neudruck mit Einleitung (9-90), Stuttgart-Bad Cannstatt 1971.

2. Hegels Vorlesungen über Rechtsphilosophie. 1818-1831.

Bd. I: Einleitung: Die 'Rechtsphilosophie' von 1820 und Hegels Vorlesungen über Rechtsphilosophie. Der objektive Geist, aus der Heidelberger Enzyklopädie von 1817 mit Hegels Vorlesungsnotizen von $1818 / 19$. Naturrecht und Staatswissenschaft, Vorlesung von 1818/19 nach der Nachschrift Carl Gustav Homeyers. Rezensionen und andere zeitgenössische Stellungnahmen zur 'Rechtsphilosophie' (1821-1833). Stuttgart-Bad Cannstatt 1973 .

Bd. II: Die 'Rechtsphilosophie' von 1820 mit Hegels Vorlesungsnotizen 182 I-I825. Stuttgart-Bad Cannstatt 1974.

Bd. III: Einleitung. Philosophic des Rechts, Vorlesung von I822/23 nach der Nachschrift von H.G. Hotho. Stuttgart-Bad Cannstatt 1974.

Bd. IV: Einleitung: Der exoterische und der esoterische Hegel (1824-1831). Philosophie des Rechts, Vorlesung von $1824 / 25$ nach der Nachschrift von K.G. v.Griesheim. Der objektive Geist, aus der Berliner Enzykopädie zweite und dritte Auflagc (1827 und 1830). Philosophic des Rechts, nach der Vorlesungsnachschrift von D.F. Strauß von 1831, mit Hegels Vorlesungsnotizen. Stuttgart-Bad Cannstatt 1974.

3. Lewis White Beck: Kants 'Kritik der praktischen Vernunft'. Ein Kommentar. Ins Deutsche übersetzt von K.-H. Ilting. München 1974.

4. Lewis White Beck: Akteur und Betrachter. Ins Deutsche übersetzt von K.-H. Ilting. Frciburg/München 1976.

5. Hegels Vorlesungen zur Religionsphilosophic.

Bd. I: Dic Vorlesung von 1821 , nach Hegels handschriftlichem Manuskript in Synopse mit der Ausgabe von 1840 . Napoli 1976.

6. G.W.F. Hegel. Naturphilosophic.

Bd. I: Die Vorlesung von $1819 / 20$ in Verbindung mit Karl-Heinz flting herausgegeben von Manfred Gies. Bibliopolis, Napoli 1982, XIX, 152 PP. 
7. G.W.F. Hegel. Die Philosophie des Rechts. Die Mitschriften Wannenmann (Heidelberg $1817 / 18$ ) und Homeyer (Berlin $1818 / 19$ ). Herausgegeben, eingeleitet und crläutert von Karl-Heinz Ilting. Klett-Cotta, Stuttgart I983, 399 pp.

(Also 17 book reviews and 10 as yet unpublished texts.) 